\title{
Fault Diagnosis for Power Grid Systems Based on Rough Set and Bayesian Network
}

\author{
Baoyi Wang ${ }^{1, a}$, Chongchong Liu ${ }^{1, b}$, Shaomin Zhang $^{1, c}$ \\ ${ }^{1}$ School of Control and Computer Engineering, North China Electric Power University, \\ Baoding, 071003, China \\ aemail: wangbaoyiqj@126.com, 'email: 740448289@qq.com, 'email: zhangshaomin@126.com
}

Keywords: Power System; Fault Diagnosis; Rough Set; Bayesian Network

\begin{abstract}
In terms of the uncertainties and incompleteness of alarm information in power grid fault diagnosis, this paper proposes a fault diagnosis method based on rough set combined with Bayesian network. Using the ability of rough set to reduce knowledge and process indeterminate information and mine fault information hierarchically, using the attribute reducing method based on cognizable matrix and information entropy, the optimal attribute reduction combination is extracted. Finally, by means of the reduction decision table formed by optimal attribute reduction combination, the Bayesian network model is built for parallel reasoning of each region, and the nodal probability is trained to achieve fault diagnosis. The experiment proves that this method can diagnose the fault rapidly and accurately, and has strong fault tolerance and adaptability.
\end{abstract}

\section{Introduction}

The method of accurate and rapid power grid fault diagnosis is of great significance to the modern power grid operation, which is an important means to guarantee the reliability of power supply[1]. Power system fault diagnosis is the process of fault identification based on various kinds of information. In recent decades, domestic and foreign scholars have put forward a series of fault diagnosis methods, including artificial neural network ${ }^{[2-3]}$, Petri net ${ }^{[4-5]}$,expert system ${ }^{[6]}$ and so on.

There are two main problems of the fault diagnosis for SOE(Sequence Of Events) alarm information: on the one hand, when a circuit breaker or relay protection device alerts false refusing action and error information transmission channel breaks, it will lead to alarm information missing or error diagnosis deviation; On the other hand, the alarm information should be dealt with after failure within a certain time interval, otherwise the fault has nothing to do with the alarm information, and it will be hard to identify the event interval[7-8].

In terms of the complexity of power grid, this method utilizes rough set theory to optimize the attributes of decision table and extract diagnosis rules, and takes advantage of Bayesian model to maximum a posterior probability of fault groups, thus getting the fault elements.

\section{Rough Set Theory}

Rough set theory is a kind of new mathematical tools to deal with uncertain and imprecise problems, and the most significant characteristic of it needs to provide no prior information out of data collection to solve the problems, such as the required prior probability in statistics and the membership degree of fuzzy focus, which can effectively analyze and deal with imprecise and incomplete data and then discover the implicit knowledge, reveal the potential regularity[9].

In rough set, we define quad $I S=(U, Q, V, F)$ as a decision table. $U$ is the theory field, and $Q$ is the set of properties, which is composed of condition attribute $C$ and decision attribute $D$, while $C \cap D=$ $\Phi, C \cup D=Q . V$ is the attributes domain set, and $V$ is equal to $\bigcup_{q \subset Q} V_{q}$ while $V_{q}$ is the range of values of q. $f: U \times Q \rightarrow V$ is the function of decision table.

Rough set theory describes the characteristics of objects by a two-dimensional decision table. According to the decision table formed by existing data, it uses the knowledge of rough set to 
remove redundant condition attributes, thus excluding redundant condition attributes from complex decision table and achieving the minimal condition attributes decision table[10].

After the reduction, the decision table will be an incomplete decision table, and it only contains those necessary condition attributes values in decision-making. But it has all the knowledge of original knowledge system so as to effectively simplify the knowledge and obtain minimal diagnostic rules. The specific algorithm steps could be considered in the references[11].

\section{Bayesian Theory}

If the event $A_{1}, A_{2}, \ldots$, and $A_{n}$ are incompatible, and these events are inevitable events, that is, $A_{1}$ $+A_{2}+\ldots+A_{n}=U$ (inevitable events). All events should satisfy:

$$
P(B)=\sum_{i=1}^{n} P\left(A_{i}\right) P\left(B \mid A_{i}\right)
$$

This is the so-called probability formula.

According to the conditional probability formula, we could deduce that:

$$
\begin{aligned}
& P\left(A_{i} \mid B\right)=\frac{P\left(A_{i}\right) P\left(B \mid A_{i}\right)}{P(B)} \\
& P(B) P\left(A_{i} \mid B\right)=P\left(A_{i}\right) P\left(B \mid A_{i}\right) \\
& P\left(A_{i} \mid B\right)=\frac{P\left(A_{i}\right) \mid P\left(B \mid A_{i}\right)}{\sum_{i=1}^{n} P\left(A_{i}\right) P\left(B \mid A_{i}\right)}
\end{aligned}
$$

The physical meaning of the Bayesian formula is that test event $\mathrm{B}$ always occur at the same time with one of all the incompatible events. If we have already known the probability $P\left(A_{i}\right)$ of an event $A_{i}$ before the test and the conditional probability $P\left(B \mid A_{i}\right)$ (i is equal to $1,2, \ldots, \mathrm{n}$ ) after a test event $B$ happens, we need to calculate the probability of each event like $A_{1}, A_{2}, \ldots, A_{n}$.

Bayesian network is a directed cyclic graph, in which each node represents a random variable, and the edge between nodes represents the direct dependency relationship between variables. Each node is attached with a probability distribution, while root node is attached with a marginal distribution and the non root node is attached with conditional probability distribution. Joint probability distribution of decomposition can reduce the complexity of the probability model, while the introduction of the Bayesian network did not further reduce the complexity, so Bayesian network is straightforward and easy modeling.

In Bayesian network, the node without directed arc input is called root node, which should be determined the prior probability; the node with directed arc input is called child node, and the the node with directed arc output is called parent node. For each child node the conditional probability under different status of the parent node should be determined.

The connection of a certain power grid system is shown in figure 1, including four regions:Sec1, Sec2, Sec3, Sec4, A, B and $C$ represent for bus lines. CB1, CB2, CB3, CB4, CB5 and CB6 represent for circuit breakers. $A_{p}, B_{p}$ and $C_{p}$ respectively represent for bus protectors.

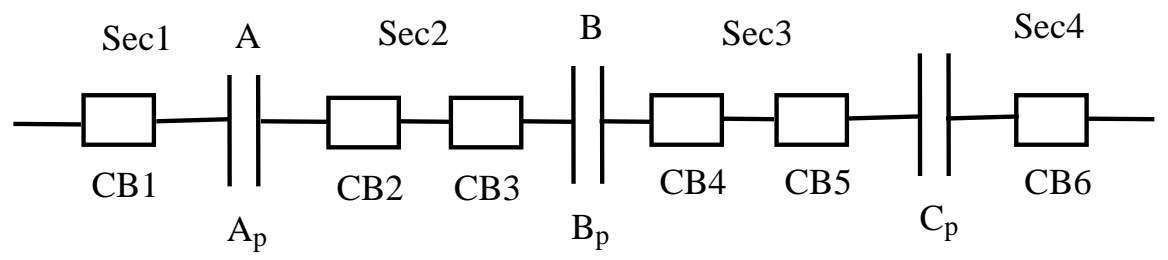

Fig.1. A Certain Power Grid

Learning from the definition of Bayesian network, we can get the Bayesian network model of the fault zone. The collection of various nodes $F$ is equal to $\{C B 1$ and $C B 2, C B 3, C B 4, C B 5, C B 6, A, B$, $C\}$. The collection of alert protection information $D$ is equal to $\{C O 1$ and $C O 2, C O 3, C O 4, C O 5$, CO6, $A_{p}, B_{p}, C_{p}$ \}. Collection $E$ represents for the casual relationship of alarm information after element fault protection. So we can get the Bayesian network model of power grid of figure 1 , 
which is shown in figure 2 .

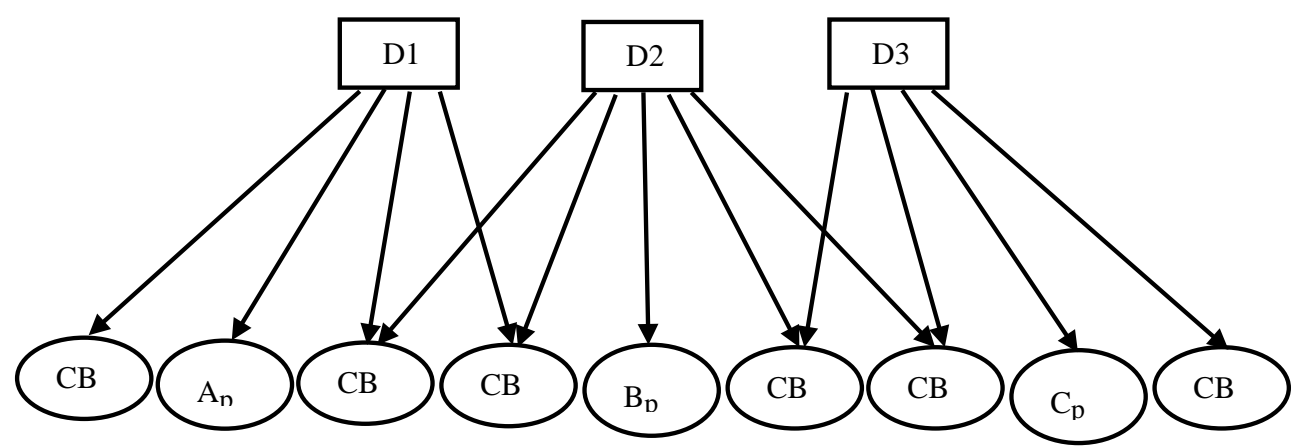

Fig.2. Bayesian Network Model

\section{Probability Calculation of Fault Hypothesis}

We use $H$ to represent for fault hypothesis, and it contains possible combinations of all the suspicious fault components. To find out which group state of suspicious components has the maximum posterior probability to explain the protection action information.

$$
\operatorname{Bel}\left(\mathrm{H}_{1}\right)=\max \alpha \prod_{j} \lambda\left(D_{j} \mid F, K\right) \prod_{i: F_{i}=F_{i}^{+}} \pi_{i} \prod_{i: F_{i}=F_{i}^{-}}\left(1-\pi_{i}\right)
$$

The $a$ in the formula is a normalization constant. $\lambda\left(D_{j} \mid F, K\right)$ is the credibility of the observed protection behavior $D_{j}$ under the condition of given fault hypothesis $H_{1}$ and all switch behaviors. $\prod_{\mathrm{i}: F_{i}=F_{i}^{+}} \pi_{i}$ is the prior probability of all the failure element sets of Fault hypothesis $H_{1}$;

$$
\prod_{\mathrm{i}: F_{i}=F_{i}^{-}}\left(1-\pi_{i}\right) \text { is prior probability of no failure element sets of Fault hypothesis } H_{1}[12] \text {; }
$$

\section{Analysis of Example}

Utilizing the proposed method, author makes experiments to analyze the example with the tool of Java and Matlab. In the figure 3 of local power grid, there are 2 bus lines, 4 transformer, 4 circuits and 3 power areas. In the local power grid, bus differential protection $(B R 1, B R 2)$ is the main bus protection. Current protection (OR1,OR2,OR3) is the backup protection of bus. Distance protection (DR1, DR2, DR3, DR4) is the protection of line $L 1$ and line L2. Current protection (OR6, OR7) is the protection of line $L 3$ and line $L 4$. Transformer differential protection $(T R 1, T R 2)$ is the main protection of transformer $T 1$ and transformer T2. Current protection (OR2, OR3, OR4, OR5) is the backup protection of $T 1$ and $T 2$. Transformer current protection (TR3, TR4) is the main protection of transformer $T 3$ and transformer T4; $C B$ represents for circuit breakers.

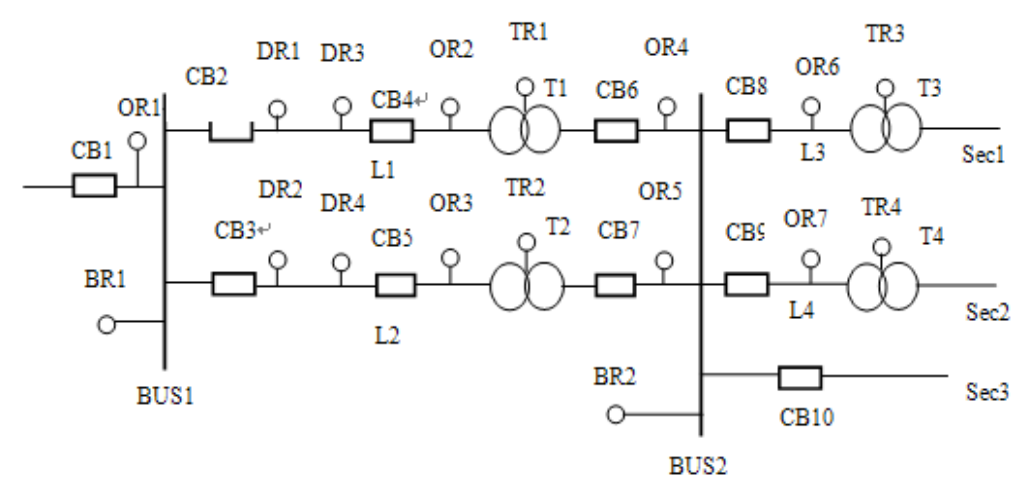

Fig.3. Local Power Grid Diagram 
According to the algorithm of attribute reduction based on differential matrix put forward by Skowron ${ }^{[13]}$, we can get the reduction decision table of all kinds of faults.

According to the 2009 protection action times[14], we can count the prior probability and use the formula of fault hypothesis to get the failure elements with the failure probability .

Preset the fault location and get the results of the diagnosis as shown in table 1 according to the fault information. When the fault information is missing or not the core attribute (such as sample 3, 6), we can get the correct diagnosis (the probability of the location of the assumed failure diagnosis is also high); When part of the core attribute information is missing or something goes wrong (sample 2), we can still get the correct diagnosis (the probability of the location of the assumed failure diagnosis is not very high); When the core attribute information is lost and other attributes information is right (sample 5,7,9), we can also get the right diagnosis (the probability of the location of the assumed failure diagnosis is low, but the failure probability of it ranks high); When some faults information is missing or wrong(sample 8), the diagnosis of fault zone with the highest probability is not the assumed fault area, but the probability of assumed fault zone is generally in the top few, thus achieving the purpose of providing the dispatcher with auxiliary decision-making.

Table.1. Power Grid Fault Diagnosis Result

\begin{tabular}{|c|c|c|c|c|c|c|c|c|c|c|c|c|c|c|c|c|c|}
\hline \multicolumn{3}{|c|}{ No Assumed location OR1 } & R2 & R3 & OR6 & $\mathrm{OR}$ & R8 & R3 & $\mathrm{DR}$ & TR & TR & ГR3 & R4 & 3R1 & BR2 & \multicolumn{2}{|c|}{ Fault Location Possibility } \\
\hline 1 & Bus1 & 0 & 0 & 0 & 0 & 0 & 0 & 0 & 0 & 0 & 0 & 0 & 0 & 1 & 0 & $\begin{array}{l}\text { Bus1 } \\
\text { NO }\end{array}$ & $\begin{array}{l}0.819 \\
0.096\end{array}$ \\
\hline 2 & Bus1 & 0 & 0 & * & $*$ & $*$ & 0 & 0 & 0 & 0 & 0 & 0 & 0 & 1 & 0 & $\begin{array}{l}\text { Bus1 } \\
\text { NO } \\
\text { L3 } \\
\text { L4 }\end{array}$ & $\begin{array}{c}0.669 \\
0.079 \\
0.079 \\
0.079\end{array}$ \\
\hline 3 & L2 & 1 & 0 & 0 & 0 & 0 & 0 & 0 & 1 & 0 & 0 & 0 & 0 & 0 & 1 & $\begin{array}{l}\text { L2 } \\
\text { Bus1 }\end{array}$ & $\begin{array}{l}0.783 \\
0.104\end{array}$ \\
\hline 4 & L2 & 0 & 0 & 0 & 0 & 0 & 0 & 0 & * & 0 & 0 & 0 & 0 & 0 & $*$ & $\begin{array}{l}\text { L2 } \\
\text { NO } \\
\text { Bus1 } \\
\text { Bus2 } \\
\text { T1 } \\
\text { T2 }\end{array}$ & $\begin{array}{l}0.238 \\
0.238 \\
0.119 \\
0.119 \\
0.119 \\
0.119\end{array}$ \\
\hline 5 & $\mathrm{~T} 1$ & 0 & 0 & 0 & 0 & 0 & 0 & 0 & 0 & 1 & 0 & 0 & 0 & 0 & 0 & $\begin{array}{l}\text { T1 } \\
\text { NO }\end{array}$ & $\begin{array}{c}0.820 \\
0.096\end{array}$ \\
\hline 6 & $\mathrm{~T} 1$ & 0 & 1 & 0 & 0 & * & 0 & 0 & 0 & 1 & 0 & 1 & 0 & 0 & 0 & $\begin{array}{l}\text { T1 } \\
\text { T3/Sec1 }\end{array}$ & $\begin{array}{c}0.794 \\
0.171\end{array}$ \\
\hline 7 & $\mathrm{~T} 1$ & 0 & $*$ & 0 & 0 & 0 & 0 & 0 & 0 & $*$ & 0 & 0 & 0 & 0 & 0 & $\begin{array}{c}\text { T1 } \\
\text { NO } \\
\text { Bus1 } \\
\text { Bus2 } \\
\text { T2 }\end{array}$ & $\begin{array}{l}0.423 \\
0.211 \\
0.106 \\
0.106 \\
0.106\end{array}$ \\
\hline 8 & $\mathrm{~T} 1$ & 0 & 1 & 0 & 0 & * & 1 & 0 & 0 & 0 & 0 & 0 & 0 & 0 & 0 & $\begin{array}{c}\text { Sec3 } \\
\text { T1 }\end{array}$ & $\begin{array}{c}0.664 \\
0.176\end{array}$ \\
\hline 9 & T4/Sec2 & 0 & 0 & 0 & 0 & 0 & 0 & 0 & 0 & 0 & 0 & 0 & * & 0 & 0 & $\begin{array}{l}\text { T4/Sec2 } \\
\text { NO } \\
\text { Bus1 } \\
\text { T1 } \\
\text { T2 }\end{array}$ & $\begin{array}{l}0.254 \\
0.254 \\
0.127 \\
0.127 \\
0.127\end{array}$ \\
\hline
\end{tabular}

According to the analysis of experiment results, we can conclude that the diagnostic method can 
help get more reasonable results. Compared with the proposed algorithm in the references[14-16], our algorithm has the following advantages:

1. With making full use of the knowledge reduction of rough set and Bayesian network pattern classification ability, this diagnosis method is of high accuracy.

2. It uses membership function to deal with the relationship between protection and elements, which dynamically expresses the process of "element fault-protection action-breaker trip-cut off the connection between the fault components and protection".

3. Use the method of Bayesian theorem to calculate the probability of diagnosis results. It enables to give the most likely failure location.

\section{Conclusion}

This paper proposes a power grid fault diagnosis method based on Bayesian network and rough set. In view of the complexity of power grid, it has strong adaptability and flexibility. Due to the combination of rough set theory and Bayesian network, this method deeply excavates alarm information knowledge. And it uses probability to analyze the wrong protection action, thus improving the diagnosis accuracy of this method under the condition of error and missing of fault alarm information. Examples show that this method has the ability to process the uncertainty of power grid fault diagnosis problems in time and space, thus improving the accuracy.

\section{Acknowledgement}

In this paper, the research was sponsored by Scientific research project of Hebei Province (Project No. Z2012077).

\section{References}

[1] LIN Sheng,HE Zheng-you,QIAN Qing-quan. Review and development on fault diagnosis in power grid [J]. Power System Protection and Control. 2010(04).

[2] XIONG Guo-jiang, SHI Dong-yuan, ZHU Lin . Fault diagnosis of power grids based on multi-output decay radial basis function neural network $[\mathrm{J}]$.Power System Protection and Control. 2013(21).

[3] HAO Jing,ZHU Feng,WANG Yun-ran,LAN Hua,XU Yun-shi,LI Lin-lin. Fuzzy PSO-BP Neural Network Method of Distribution Network Fault Line[J]. Electrical Measurement \& Instrumentation. 2011(12).

[4] LAN Hua,LI Jin,GAO Ao,YANG Jian-biao. Fault Diagnosis of Distribution Networks by the Regional Parallel Reasoning Based on Rough Set and Petri Nets[J]. Electrical Measurement \& Instrumentation. 2012(01).

[5] WU Jin-fu,RU Yuan,MENG Zhen,LI Shu-guang,RU Feng,JU Yong-feng. Research on Fault Diagnosis System of Power Grid Based on Petri Net[J]. Modern Electronics Technique. 2011(21).

[6] JI Yong-Cheng. Research and Implementation on Diagnosis of Power Network Expert System Based on Fuzzy Identification[D]. University of Ji Lin. 2011.

[7] LIU Xiaoqin;WANG Dazhi;ZHANG Cuiling;JIANG Xuechen;NING Yi. Fault Diagnosis Approach of Power Grid Based on Genetic Wavelete Neural Network[J]. Journal of Petrochemical Universities. 2013(6).

[8] GAO Zhen-xing,GUO Chuang-xin,YU Bin,LUO Yu-hai,PENG Ming-wei,YANG Jian. Study of a fault diagnosis approach for power grid with information fusion based on multi-data resources [J]. Power System Protection and Control. 2011(6). 
[9] WANG Lin-xian,LI Nai-yong. A power network fault diagnosis method based on rough set theory and fault information networks [J].RELAY , 2008, 36(8): 59- 63.

[10] CHEN Ding-san,YANG Hui-zhong. Multiple Models Soft-sensing Technique Based on An Improved Weighted Rough Set[J]. Control and Instruments in Chemical Industry, 2010,37(1):11-15.

[11] WU Xin, GUO Chuang- xin, CAO Yi-jia. A New Fault Diagnosis Approach of Power System Based on Bayesian Network and Temporal Order Information[J]. Proceedings of the CSEE, 2005, 25(13):14-18.

[12] ZHU Yu-xiang, WU Qian, WANG Yun-ran, LI Jia-bin, LAN Hua. rough set and Temporal Bayesian Networks Based Fault Diagnosis for Power Systems[J]. Electrical Measurement \& Instrumentation, , 2013, 50(11): 65-68.

[13] Skowron A. Intelligent decision support handbook of application and advances of the rough set theory [M]. Holang: Kluwer Academic Publishers, 1992.

[14] SHU Hong-chun, SUN Xiang-fei, SI Da-jun. A Study of Fault Diagnosis in Distribution Line Based on Rough Set Theory[J]. Proceedings of the CSEE. 2001(10).

[15] Zhao Dongmei, Han Yue, Gao Shu. New Reduct Algorithm of Decision Table for Electric Power Grid Fault Diagnosis[J]. Automation of Electric Power Systems. 2004(04).

[16] LI Ran,LI Jing-hua,LI He-ming. Fault Diagnosis Layer Model of Distribution Network Based on Weighted Mean Roughness [J].Power System Technology. 2006(02). 